\title{
The WSN Coverage Optimization of the Diversified AFSA Based on Chaos Learning Strategy
}

\author{
Yongqiang $\mathrm{Li}^{1}$ and PanJin ${ }^{2}$ \\ ${ }^{1}$ (Software Technology Vocational College, North China University of Water \\ Resources and Electric Power, Zhengzhou 450045, China) \\ ${ }^{2}$ (Henan Procuratorial Vocational College Zhengzhou 451191, China) \\ 1696450309@qq.com,1632921040@qq.com
}

\begin{abstract}
WSN coverage optimization is an important problem. Considering that the artificial fish algorithm is easy to fall into local optimum and of slow convergence, an improved algorithm has been proposed in this paper. The chaos strategy is used to carry out the initialization of the foraging behavior, which makes the fish swarm evenly distributed in space, to avoid the randomness of the initialized indiv dual. At the same time, the concept of diversity is introduced in the swarm behaviox which makes its avoidance of congestion further improved. Through standard test functions and simulated network coverage testing, the algorithm presented in this paper improves the WSN network node coverage, which effectively reduces the network cost gnd further improves the network coverage optimization.
\end{abstract}

Key words: chaos; AFSA; diversity; wireless sensing

\section{Introduction}

Wireless Sensor Net thork (WSN) is a new information technology developing with the progress of communication technology and embedded technology. Coverage optimization has always been an important aspect of the wireless sensing. The quality of the optimized result directly influences whether the network resource is used reasonably. It is a key problem in wireless sensing bow to reasonably keep the network coverage and extend the network life cycle.

Wireless sensing is mainly made up of multiple separate nodes, and these nodes are divided into static and dynamic nodes. Between the nodes, the environmental interactions are implemented through sensing and controlling parameters. The static nodes are usually deployed artificrally and suitable for some safe zones, because this way can be adjusted according to the position of the scene, which can realize good coverage. The dynamic node way is to deploy randomly, easy to form some blind areas and overlapping areas. At presen, the research on WSN has made some achievements. The reference [1] presents an optimizing strategy based on the particle swarm algorithm, and effectively prevents the algorithm from falling into the premature trap through introducing the disturbance factor, which accelerates the algorithm convergence. It is verified by simulation experiments that the optimization algorithm can effectively improve the performance of network coverage. In addition, it is compared with the latest algorithm. The reference [2] presents a three-dimensional particle swarm algorithm (3D - PSO), which can improve the execution efficiency and positioning accuracy of the algorithm. It limits the searching space of particles, to speed up the convergence rate of the positioning results. The simulation results show that the algorithm has better robustness and higher positioning precision. The reference [3] constructs a probability Voronoi model from the perspective of multiple sensors collaborative monitoring, trying to solve the above problems. And the validity of the model has been verified with the biggest breakthrough path algorithm based on the probability Voronoi model. The model is put forward for the first time and worth 
popularizing in practice. The reference [4] puts forward a kind of WSN coverage optimization algorithm based on genetic PSO. The maximum coverage of the wireless sensor is used as the objective function. By using the genetic algorithm with self-adaptive crossover and variation factor, search the solution space. The strong global search capability of PSO is used to increase the search scope, to make particle coverage more efficient and to strengthen the optimum searching ability of the algorithm. It improves the coverage of nodes and solves the problem of premature. The simulation results show that the method can effectively realize the WSN coverage optimization. The reference [5] describes the solution of the problem that the current coverage algorithm consumes too much energy on nodes and is poor in monitoring accuracy when used in monitoring spatially continuous distribution of physical quantity. In this paper, a new WSN coverage algorithm based on the dynamic nested grid technology in combination the numerical analysis of hydromechanics (DNGCA), and the simulation results show that the algorithm is improved much on the main coverage indicators compared to other algorithms. The reference [5] puts forward a WSN coverage algorithm of particie swarm. It uses the maximum coverage of the wireless sensor as the objective function, with several particle swarms independently searching the solution space, to increase the search area of the particles and reduce the possibility of local optimum. The evolutionary particle is used to make the particle coverage more efficient, which inhproves the optimum searching capability of the algorithm, effectively avoids the "premature" problem easy to occur in the standard particle swarm algorithm and improves the stability of the algorithm. The reference [7] argues to optimizing the WSN nodes by ysing AFSA and particle swarm algorithm. Due to their shortcomings, the particle warm algorithm is easy to fall into local optimum, which is difficult to achieve the global convergence; the genetic algorithm in different wireless sensing strueture has to determine the methods of genetic manipulation, so the solving process is veryecomplex; the ant colony algorithm is strong in local search, but because of its low speed in solving in the early stage, it covers the timeliness of optimization

AFSA (AFSA) [G] is a new intelligent and bionic algorithm presented by simulating the foraging and livingactivities of fish, which is easy to implement but slow in convergence and easy to be premature. On the basis of this, an improved AFSA is proposed in this paper. In the init alization phase of artificial fish, the chaos reverse learning strategy is used, which enables the fish swarm initialized to be evenly distributed in space, to speed up the global convergence of the algorithm. The simulation experiment shows that compared with the AFSA, the algorithm of this paper has a high accuracy of optimum searching and a high speed of convergence.

\section{The Network Coverage Model}

\subsection{Node Coverage Description}

In the model, set a target area, which is divided into $M \times N$ grids. In this area, place a set number of fixed nodes and mobile nodes and set the coordinate of each node. The perception radius and the communication radius of a node is set as $r$ and $R$, respectively. The node collection of the sensor is represented as $C=\left\{c_{1}, c_{2} \ldots \ldots c_{n}\right\}$, and $c_{i}=\left\{x_{i}, y_{i}, r\right\}$ means that the circle center is $\left(x_{i}, y_{i}\right)$ and the radius is $r$. 


\subsection{The Description of Coverage}

If the coordinate of the node $C_{i}$ in the sensor network is $\left(x_{i}, y_{i}\right)$, and the coordinate of any node ${ }^{T_{j}}$ in the area is $\left(x_{j}, y_{j}\right)$, the distance between the network node and the target node is as follows:

$$
L\left(C_{i}, T_{j}\right)=\sqrt{\left(x_{i}-x_{j}\right)^{2}+\left(y_{i}-y_{j}\right)^{2}}
$$

Wherein, ${ }^{\lambda_{1}}$ and ${ }^{\lambda_{2}}$ are the input parameters and the measured probability from node $C_{i}$ to the target node ${ }^{T_{j}}$ is:

$$
P_{\left(C_{i}, T_{j}\right)}= \begin{cases}0 & R_{s}+R_{e} \prec L\left(C_{i}, T_{j}\right) \\ e^{\frac{-2 \lambda_{1} \alpha}{\lambda_{2} \beta}} & R_{s}-R_{e} \leq L\left(C_{i}, T_{j}\right) \leq R_{s}+R_{e} \\ 1 & R_{s}-R_{e} \geq L\left(C_{i}, T_{j}\right)\end{cases}
$$

\section{A Brief Introduction of the AFSA}

The AFSA (AFSA) is a kind of intelligent agorithm, which is set in the search space and uses $n$ artificial fish. The state of number $i$ artificial fish is represented as the vector $X_{i}=\left(X_{i 1}, X_{i 2}, X_{i 3}, \ldots \ldots . . X_{i N}\right)$ and the curnentood density of the artificial fish is represented as $Y=T(x)$. In the aftificial fish swarm, the distance between a fish and another is expressed as: ${ }^{d_{i j}}=\left\|x_{i}-x_{j}\right\|$ and ine step length in the artificial fish swarm is represented as step. The view range of the artificial fish swarm is Range, and Number is used to represent the individual number of the fish swarms. In the algorithm, the state of each artificial fish is a solution. Substitute $X_{i}$ into the optimization function, to judge the quality of each fish by the value of the function. The basic behavior of the artificial fish swarm is described as follows.

\subsection{Foraging Behavior}

If the current state of the AFSA is ${ }^{x_{i}}$, according to the formula (3), randomly select a state ${ }^{x_{j}}$ with the food density meeting the condition of $T\left(x_{i}\right)<T\left(x_{j}\right)$, and then calculate by the formula (4). If the food density meets $T\left(x_{i}\right) \geq T\left(x_{j}\right)$, in the visible range, randomly select a state, where rand represents a random number.

$x_{j}=x_{i}+e^{\frac{x_{j}}{x_{i}}}$

$x_{j}=x_{i}+\operatorname{rand} \square \sum_{i=1}^{n}\left(X_{i}-\bar{X}_{i}\right)^{2}$

\subsection{Swarm Behavior}

The swarm behavior is used to avoid congestion. It is to search the number of partners currently in the visible range ${ }^{n}$ and the center position ${ }^{x_{c}}$. When the food density 
is $T\left(x_{i}\right)<T\left(x_{c}\right)$, it shows that there is higher food density at the partner's location. And then use the formula (3) to replace ${ }^{x_{i}}$ with $x_{c}$ to go to the partner's location.

\subsection{Rear-ended Behavior}

The rear-ended behavior means that the fish swarm explores the optimal artificial fish in the visible area, and when the state is $T\left(x_{i}\right)<T\left(x_{\max }\right)$ it shows that at the location of $x_{\max }$ there is a high density of food. With formula (4), use ${ }^{x_{\max }}$ to replace ${ }^{x_{i}}$. After judging, it will continue to implement the foraging behavior.

\section{The Improved AFSA}

\subsection{The Chaos Learning Strategy}

The foraging behavior of AFSA is very important. The quality of foraging behavior initialization directly influences the algorithm's speed of globa convergence and the quality of the corresponding solutions. Usually, due to the laek of some information, the random initialization method is often used to generate the initial solution of the algorithm. The reference [8] presents a chaos initialization method on the basis of the research on particle swarm algorithm and uses the strategy to initialize the AFSA, with the specific steps shown as follows:

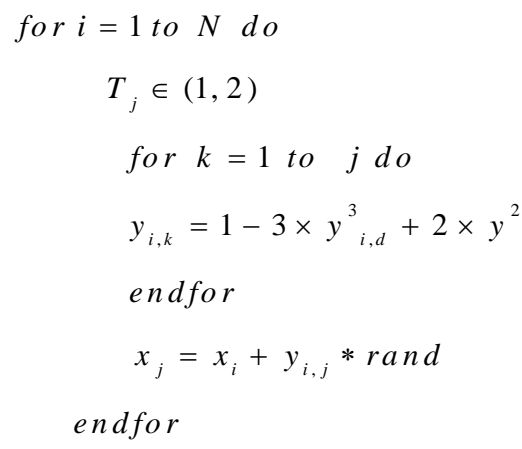

\subsection{The Dynamic Self-adaptive Swarm}

In the swam behavior, the swarm will gather at the location with high food density, which is easy to cause certain blocking situations. In settling the problem of WSN coverage, it will cause local optimum. To solve such problems, an updating strategy is presented in reference [9] in this paper. This strategy introduces the concept of diversity to the formula [4], to improve the searching ability of the algorithm.

Set the number of fish swarms in the AFSA as ${ }^{N}$ and the number ${ }^{j}$ individual in the number $i$ group of artificial fish ${ }^{x_{i}}$ as $x_{i j}=\left(x_{i j 1}, x_{i j 2}, \ldots \ldots x_{i j n}\right)$, in which $n$ is the number of dimensions, and the optimal position of the number ${ }^{j}$ generations of individual artificial fish swarm is $y_{i}=\left(y_{j 1}, y_{j 2}, \ldots \ldots . . y_{j n}\right)$. Join the current position of the fish swarm with the generations of optimal positions, written as $z_{j}=\left(x_{i j 1}, x_{i j 2}, \ldots \ldots x_{i j n}, y_{j 1}, y_{j 2}, \ldots \ldots . y_{j n}\right)$. All the individuals ${ }^{z_{j}}$ in the AFSA forms an $N \times n$ matrix $z$, which is normalized to get the following matrix $z$ 


$$
z^{\prime}=\frac{\sum_{i=1}^{n}\left(X_{\max }-\bar{X}_{\min }\right)^{2}}{\sum_{i=1}^{n} X_{\max } Y_{\text {min }}}
$$

The diversity of the fish swarm is represented as follows:

$$
F=\frac{\sum_{i=n+1}^{N} Q(i)}{\sigma_{i}}
$$

So the improved formula is

$$
x_{j}=x_{i}+r_{i j}(k) \square \text { rand } \square \sum_{i=1}^{n}\left(X_{i}-\bar{X}_{i}\right)^{2}
$$

\subsection{Steps for Coverage Optimization}

Step 1: initialize the WSN, according to the scale of the network, to set the size of the artificial fish swarm as ${ }^{N}$, the biggest mobile step length as step , visible range as Visual and the iteration as number.

Step 2: Calculate the food density of the current positions of individual fish swarms, choose those with high food density to shor on the bulletin board

Step 3: In the foraging behavior, adopt chaos learning strategy proposed in this paper to carry out initialization

Step 4: As for the rear-ended behavior and swarm behavior of the artificial fish simulated fish swarm, in swarming the impreved formula is used to select a new location.

Step 5: After one traversal of the artificialfish swarm, compare the food density in the current position with that shown on the bulletin board. If the comparison result is the former is greater than the latter, the position of the fish swarm shown on the bulletin board will be replaced.

Step 6: If it meets the end condition, input the $T(x)$ value in the bulletin board, which is the optimal scheme of WSN coverage.

\section{Analysis of the Algorithm Results}

To test the performance of this algorithm, there are two parts of tests in this paper, one of which is to test the performance of the algorithm and the other of which is to test the network coverage optimization.

\subsection{Algorithm Performance Test}

This algorithm selects three benchmark functions to test, C\# of Visio Studio.Net 2007 as the test platform, and Windows XP as the operation system. The three functions are as follows:

$$
f 1(z)=\sum_{i=1}^{D}\left[z_{i}^{2}-10 \cos \left(2 \pi z_{i}\right)+10\right]
$$

minimizes when $z_{i}=0(i=1,2, \ldots . D)$. 


$$
f 2(z)=\frac{\sum_{i=1}^{n} \cos ^{4}\left(x_{i}\right)-2 \prod_{i=1}^{n} \cos ^{2}\left(x_{i}\right)}{\sqrt{\sum_{i=1}^{n} i x_{i}^{2}}}
$$

is also a multimodal function which reaches the global minimum value 0 when $z_{i}=0(i=1,2, \ldots . . D)$.

$$
f 3(z)=418.9283 \times D-\sum_{i=1}^{D} x_{i} \sin \left(\left[x_{i}\right]^{1 / 2}\right)
$$

is a uni-modal function which

reaches the global minimum value 0 when $z_{i}=1(i=1,2, \ldots . . D)$.

On the three functions mentioned above, the Particle Swarm Algorithm, the AFSA and the algorithm presented in this paper are compared. In the AFSA, let the scate of the artificial fish swarm be $\mathrm{M}=20$, visual 2.85 , step $2.5, d 0.618, L$ max 40 and the dimension of the test function be 2 . In the Particle Swarm Algorithn, letw be 0.5, and $c 1, c 2$ are also 0.5 . Table 1 shows the results of the three algorithms.

Table 1. The Optimum Searching Results of the Four Algorithms

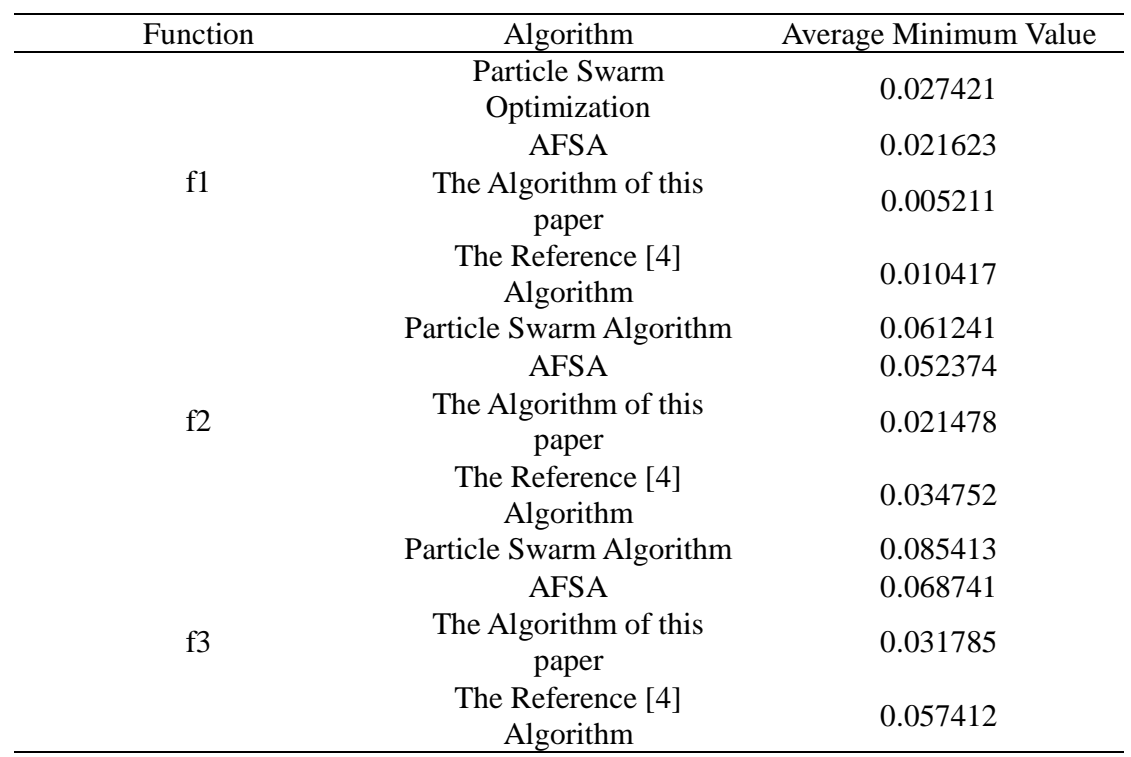

It can be seen from the results of Table 1 that the average minimum values and minimum values of the algorithm of this paper are better than that of the other three algorithms, and the test effect is ideal.

\subsection{Analysis of the Results}

\subsubsection{Wireless Sensing Coverage Test}

Set the environment of simulation experiment as: the network detection area is $10 \mathrm{~m} * 10 \mathrm{~m}$. For the nodes of each wireless sensor, the perception radius is $0.5 \mathrm{~m}$, and the communication radius is $2 \mathrm{~m}$. Under the environment of Matlab7.0, use the Core i3 computer the frequency of which is $2.2 \mathrm{GHZ}$ to carry out the simulated network coverage optimization. Let the number of artificial fish swarms be Number $=200$, the Range of artificial fish swarms be 5, the largest iteration be $N=500$, to compare the algorithm of this paper with the basic particle swarm algorithm, artificial fish algorithm and the reference [4] algorithm and make analysis. As shown in Figure 1-2. 


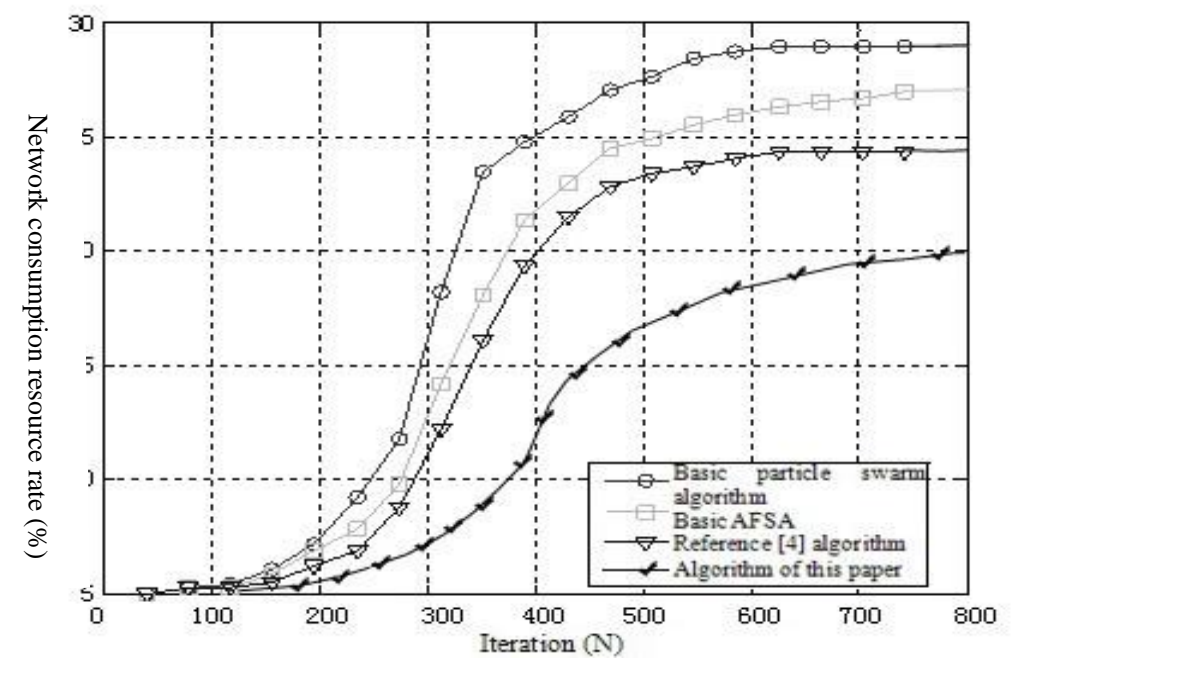

Figure 1. The Comparison of the Network Resources Consurned by the Four Algorithms

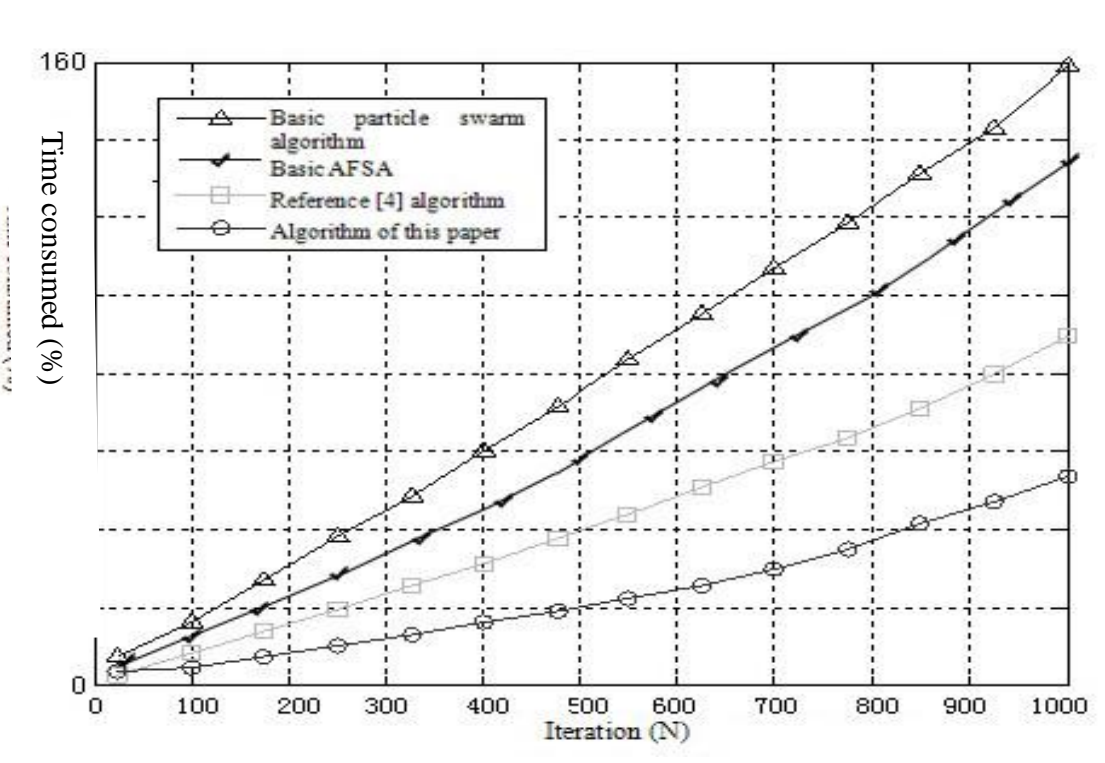

Figure 2. The Comparison of the Time Consumed by the Four Algorithms

It can be seen from Figure 1-2 that the algorithm in this paper is better than the other three/algorithms in the consumption of time and network fees, and it is found that the response time tends to be flat with the increase of the iteration, which suggests that by improving the artificial fish algorithm, the convergence has been accelerated and the optimal solution can be found by fewer iterations. Compared with other algorithms, its response time is shortened, and the aspects like stability are improved substantially.

\subsubsection{The Comparison of Other Coverage Optimization Algorithm}

In the same simulation environment, the method of random distribution is adopted to compare the Particle Swarm Algorithm, Ant Colony Algorithm, AFSA and the algorithm of this paper by experiments. The comparison results are shown in Figure 3. 


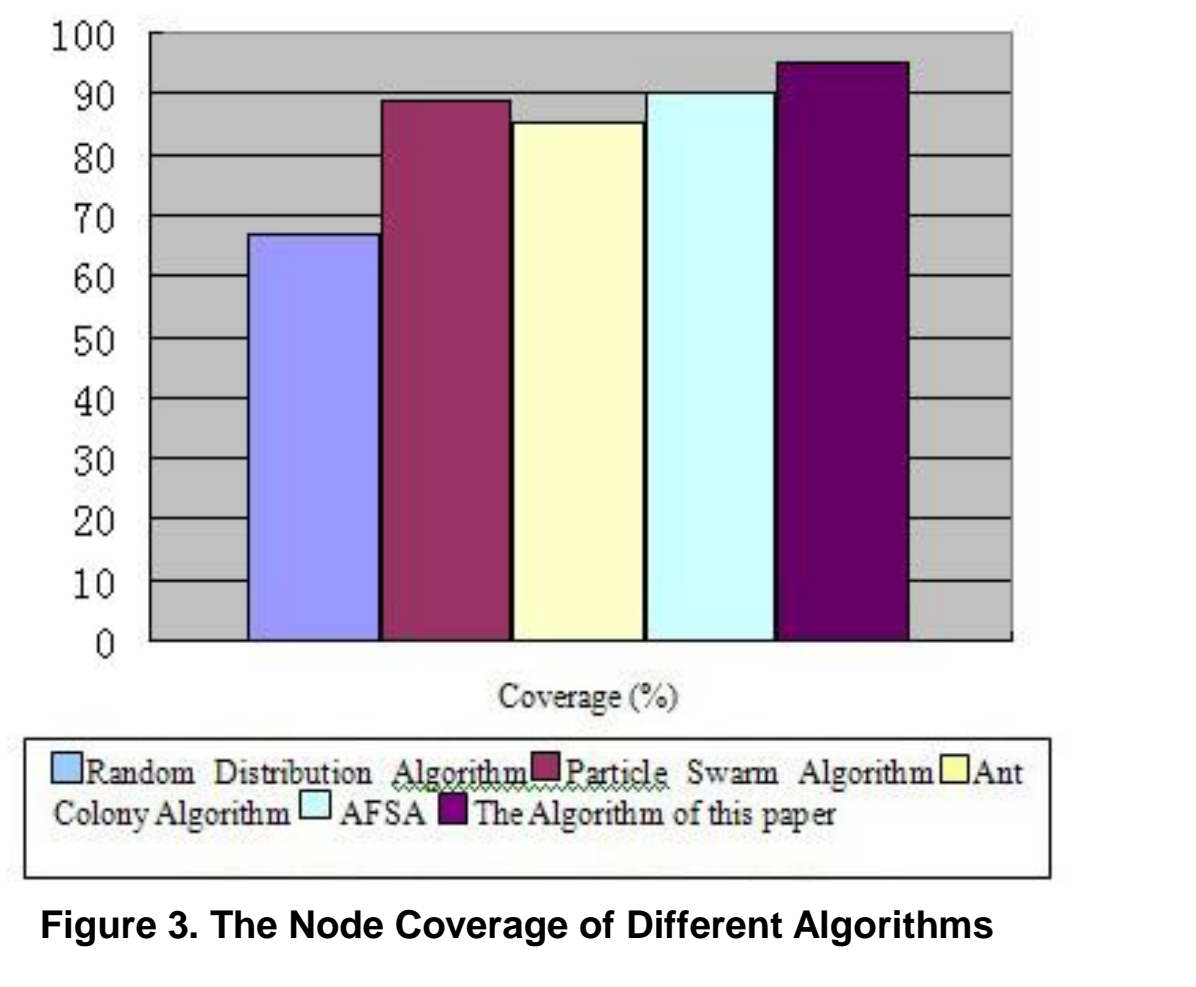

From Figure 3, it can be seen that the overage of the Random Distribution Algorithm is around $70 \%$, which cannot meet the actual needs of the WSN. The node coverage of the Particle Swarm Algorithm and the Ant Colony Algorithm ranges from 80\% to 90\%. The node coverage of the modified AFSA reaches more than 90\%, which realizes the coverage optimization in WSN and can keep high network coverage in a relatively long period to extend the network life ercle, with a good practical application effect.

\section{Conclusion}

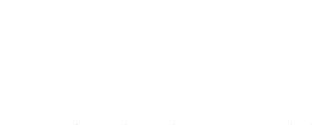

Network coverage is the key problem in sensor networks. As the AFSA is easy to fall into local optimum and has a low rate of convergence, to better optimize, based on the original research resolts, in this paper, the chaos reverse learning initialization is introduced into the algorithm. It prevents the algorithm from falling into local optimum, and at the same time introduces the concept of diversity into the improved algorithm, to accelerate the convergence rate. Through the test of the standard functions, this algorithm is better than the AFSA in each aspect. The simulation experiment shows that the improved AFSA can get the optimal coverage node at a little expense, to reduce the network delay, improve the coverage of the nodes and decrease the number of mobile nodes. It has great significance for the actual application.

\section{References}

[1] X. Xiang, "The Hybrid WSN Coverage Optimization Based on the Particle Swarm Algorithm [J]", Computer Application Research, vol. 27, no. 6, (2010), pp. 2273-2275.

[2] J. Lv, "A Study of the 3D WSN Localization Algorithm Based on the Particle Swarm Optimization [J]", Journal of Shandong University (Science Edition), vol. 48, no. 5, (2013), pp. 78-82.

[3] X. Wang, "Research on the Probability Voronoi Model and Algorithm in the WSN Coverage [J]", Journal Of Sensing Technology, vol. 25, no. 5, (2012), pp. 702-705.

[4] H. Shen, "Research on the WSN Coverage Optimization Algorithm Based on Genetic PSO [J]", Journal of Microelectronics and Computers, vol. 30, no. 3, (2013), pp. 148-151.

[5] J. Gu, "Research on the WSN Coverage Algorithm and Simulation [J]", Computer Simulation, vol. 27, no. $9,(\mathbf{2 0 1 0})$, pp. 146-148. 
[6] X. Li, Z. Shao and J. Qian, "An Optimum Searching Mode Based on Animal Commune: Fish Swarm Algorithm [J]", Journal of Systems Engineering Theory and Practice, vol. 22, no. 11, (2002), pp. 32-38.

[7] X. Yao, Y. Zhou and Y. Li, "Artificial Fish Swarm Mixed with Particle Swarm Optimization Algorithm [J]", Computer Application Research, vol. 6, (2010), pp. 2084-2086.

[8] B. Liu, L. Wang and Y. H. Jin, et al. "Improved Particle Swarm Optimization Combined with Chaos [J]", Chaos, Solitons and Fractals, vol. 25, no. 2, (2005), pp. 1261-1271.

[9] D. Zhang, Z. Guan and X. Liu, "Adaptive Partical Swarm Optimization Algorithm with Dynamically Changing Inertia Weight [J]”, Control and Decision, vol. 23, no. 11, (2008), pp. 1253-1257.

\section{Authors}

Li Yongqiang (1974.1-) Lecturer, Master, Research Orientation: Computing Network;

Pan Jin (1974.11-) Associate Professor, Master, Research Orientation: Computer Network

为方便组委会联系，请提供 2 位作者信息。

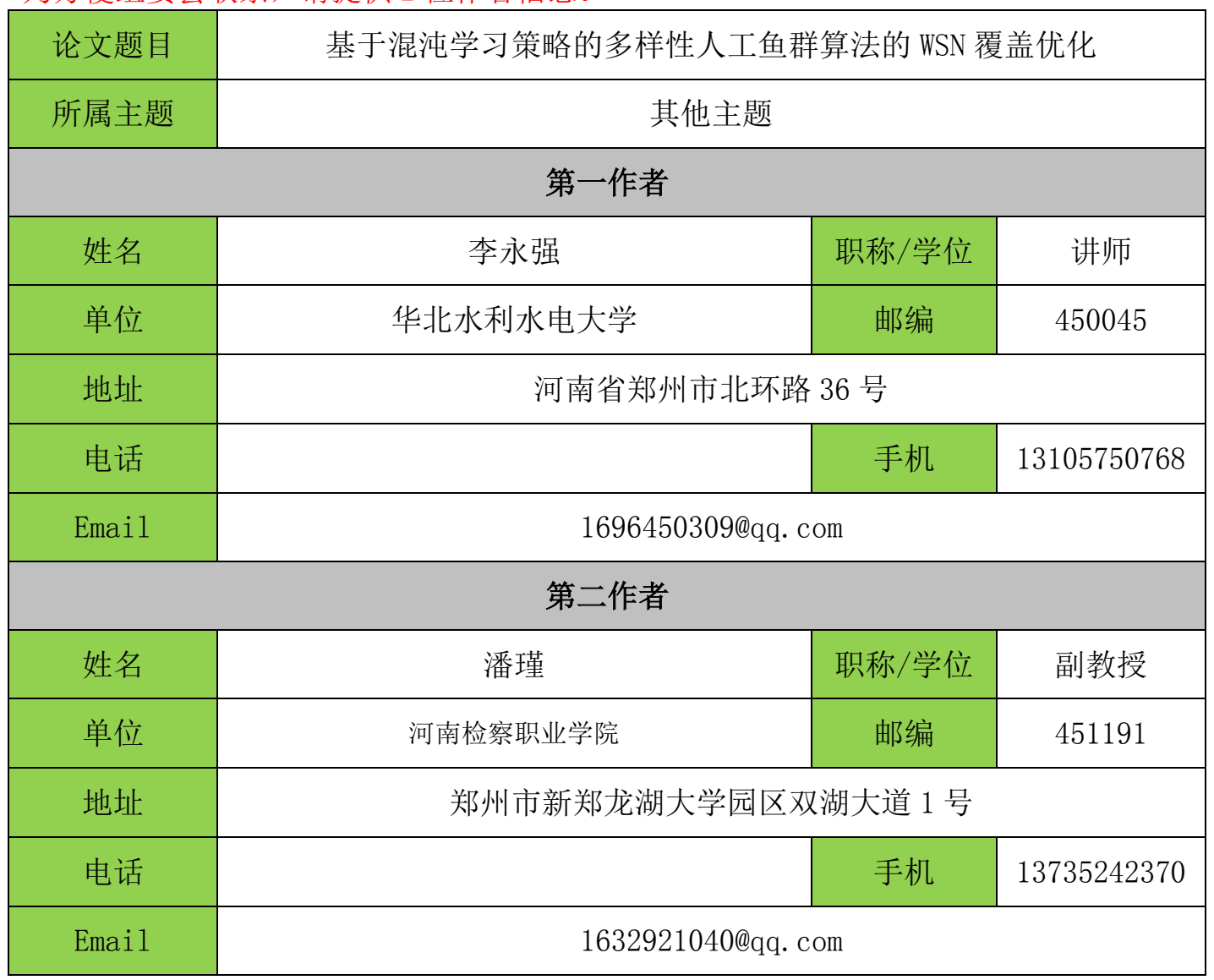


International Journal of Multimedia and Ubiquitous Engineering

Vol.9, No.8 (2014)

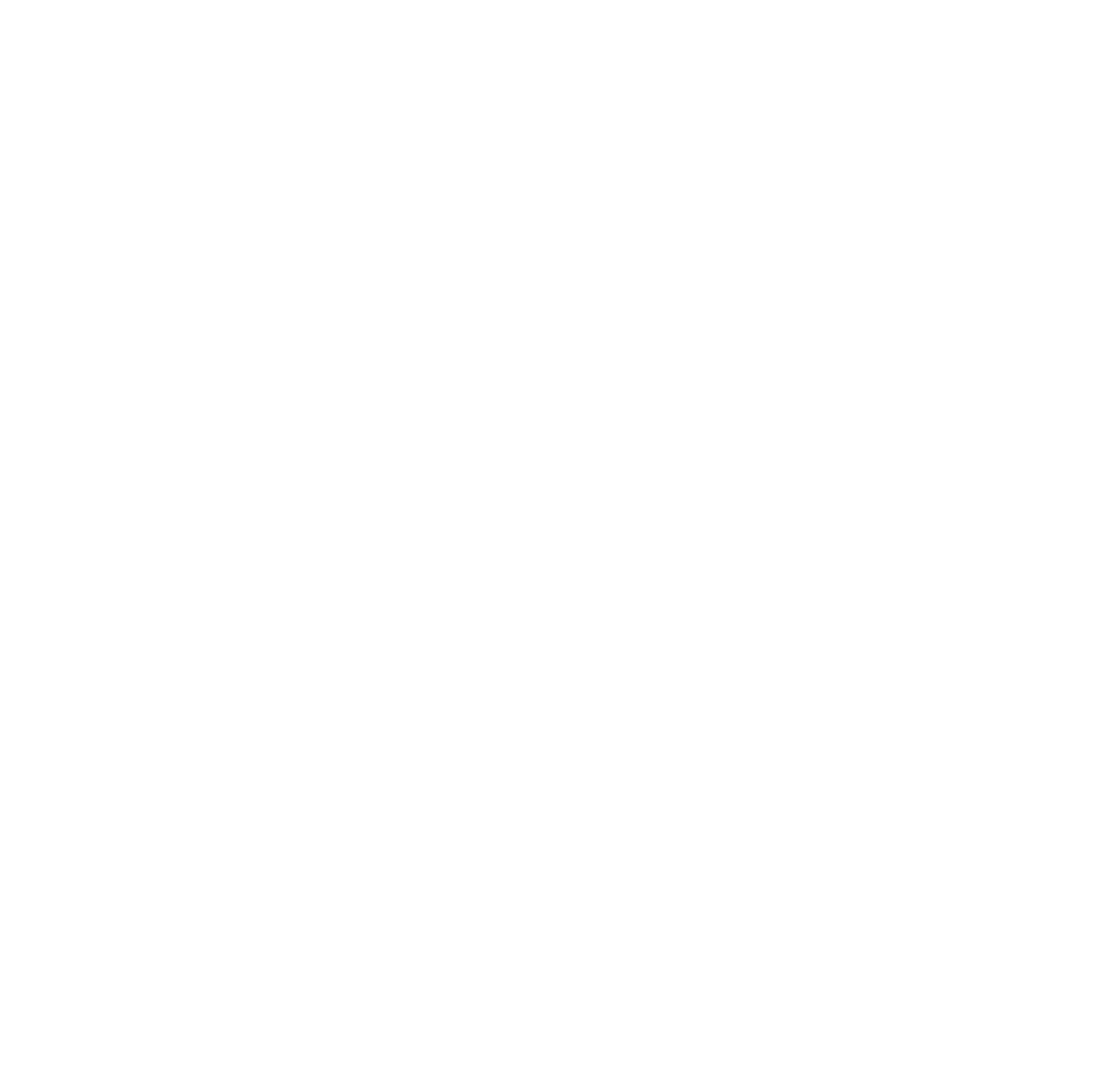

